\title{
Le fistole artero-venose protesiche: focus infermieristici
}

\author{
Milena Bosatta
}

UOC di Nefrologia e Dialisi, Ospedale Sant'Anna-Como, ASST-Lariana, Como

\begin{abstract}
Prosthetic artero-venuous fistula: the nursing focus
Prosthetic Artero-Venus fistula (PAV, also known as AVG) is a biocompatible synthetic device that connects arterial and venous segments creating an optimal vascular access for hemodialysis. New biocompatible materials withstanding barotrauma and repeated cannulations during dialysis sessions, fuel the discussion regarding the use of Prosthetic Artero-Venus fistula in patients on maintenance dialysis. The aim of this brief article is to provide health care professionals with a practical manual about how to manage this type of vascular access in daily care.
\end{abstract}

Keywords: Accesso vascolare, Complicanze, Dialisi, Fistola artero-venosa, Protesi



Milena Bosatta

\section{Introduzione}

La Fistola Artero-Venosa protesica (FAVp) consiste nell'interposizione di una protesi vascolare sintetica in materiale biocompatibile che collega una arteria a una vena e fornisce un accesso vascolare idoneo al trattamento emodialitico. Viene allestita quando una Fistola Artero-Venosa nativa (FAVn) sia ritenuta tecnicamente impossibile o non riesca a maturare. Infatti, la scelta della realizzazione di una FAVp avviene nel caso di assenza di vene superficiali adeguate (quindi la possibilità di accedere al circolo venoso profondo), obesità (soprattutto nel sesso femminile), vasculopatie periferiche (diabete mellito, collagenopatie, aterosclerosi) oppure precedenti interventi di allestimento vascolare che abbiano esaurito il patrimonio vascolare superficiale del paziente. La disponibilità di vari materiali protesici e la popolazione dialitica con avanzata età anagrafica concomitante a diverse comorbidità ha riproposto l'uso delle FAVp in alternativa al posizionamento di cateteri permanenti.

Accepted: November 8, 2016

Published online: November 24, 2016

Indirizzo per la corrispondenza:

Milena Bosatta

UOC di Nefrologia e Dialisi

ASST-Lariana Ospedale San'Anna

Via Ravona 20

22020 San Fermo Della Battaglia (CO)

milena.bosatta@asst-lariana.it
Condizioni generali e logistiche per la realizzazione delle FAVp

Le condizioni generali per la realizzazione di una FAVp comprendono la valutazione complessiva del paziente (età, comorbidità), le condizioni del Centro di dialisi di provenienza - in termini di competenza e organizzazione per la gestione di tale accesso - e la presenza di un necessario interscambio di informazioni e collaborazione tra il centro confezionatore e il centro utilizzatore. Infine, è necessario prevedere gli opportuni controlli clinici della FAVp (per esempio, attraverso un EcoColor Doppler (ECD) anche mensile) e disporre di un reparto di Radiologia interventistica per la gestione tempestiva delle eventuali complicanze della FAVp, trombosi o stenosi, necessitanti per esempio di angiografia percutanea (PTA).

Le condizioni per l'allestimento di una FAVp dipendono da un adeguato afflusso (inflow) arterioso e da un efflusso (outflow) venoso (1). L'inflow arterioso è strettamente correlato al diametro dell'arteria utilizzata per la costruzione dell'anastomosi, dal flusso ematico e dalla pressione arteriosa. L'outflow venoso, invece, è legato al diametro della vena di scarico della FAVp e dall'assenza di stenosi e trombosi venose centrali. Infine, cute e sottocute devono essere integri poiché dovranno resistere alle tensioni indotte dalla protesi e dalle ripetute incannulazioni.

In base ai vasi arterioso e venoso anastomizzati si distinguono diversi tipi di FAVp. Nella Tabella I, vengono riassunti i principali tipi di FAVp comunemente allestite (National Kidney Foundation, 2006) (2).

\section{Vantaggi e svantaggi delle fistole protesiche}

I vantaggi del confezionamento di una FAVp rispetto alla FAVn sono rappresentati dalla rapidità di esecuzione dell'impianto, dal rapido utilizzo dopo l'impianto (24 ore dal 
TABELLA I - Tipi di FAVp comunemente confezionate

\begin{tabular}{lll}
\hline Sede & Tipo & Vasi anastomizzati \\
\hline Avambraccio & Retta & Radio-Cefalica (arteria radiale-vena cefalica) \\
& Loop (circolare) & Radio-Cefalica (arteria radiale-vena cefalica) \\
Braccio & Retta & Omero-Ascellare (arteria omerale-vena ascellare) \\
& Loop “capovolto" (circolazione anti-oraria) & Omero-Basilica (arteria omerale-vena basilica) \\
& Loop & Axillo-Ascellare (arteria ascellare-vena ascellare) \\
\hline
\end{tabular}

TABELLA II - Esame obiettivo IPA della FAVp

Ispezione

Palpazione

Auscultazione
Segni di infezione, Segni di flogosi (calor, rubor, dolor, tumor), ematomi, edema, pseudoaneurismi

Verificare la presenza del fremito che è lieve o nullo sulla protesi ma presente a livello della anastomosi venosa e arteriosa

Con l'uso del fonendoscopio verificare l'intensità e le caratteristiche del fremito confezionamento per le protesi con materiali di ultima generazione) e dal collegamento di vasi venosi e arteriosi distanti e profondi tra loro e altrimenti non utilizzabili.

La nuova generazione di protesi con membrana auto-sigillante assicura un ridotto sanguinamento sui punti di sutura e di puntura e la riduzione della possibilità di formazione di pseudo-aneurismi.

Permangno invece i già noti svantaggi caratterizzati dall'aumentato rischio di trombosi, infezioni e stenosi, da una minore durata rispetto a una FAVn, da un'incidenza maggiore di casi di Sindrome da furto, dovute sia alla dinamica dell'accesso protesico che dalle condizioni anatomiche del paziente, successive a precedenti interventi vascolari o dalla presenza concomitante di sovraccarico cardiaco destro.

\section{Puntura della fistola protesica}

Prima di effettuare la puntura della FAVp è necessario seguire il percorso indicato nella Tabella II. Con l'acronimo IPA (Ispezione, Palpazione, Auscultazione) si riassumono le principali manovre da eseguire prima della puntura della protesi.

Come suggerito dalle Linee guida Kidney Disease Outcome Qualitative Initiative (KDOQI) del 2006 (2), per la puntura della FAVp è necessario controllare:

- posizione e forma della protesi (loop o retta);

- pervietà della protesi;

- direzione del flusso ematico;

- descrizione dell'intervento che specifica il tipo di anastomosi che è stata realizzata;

- stato della cute nelle aree di puntura (Tab. II);

- adeguata disinfezione:



$3^{\infty}$

Fig. 1 - Rappresentazione grafica esemplificativa di protesi punta secondo la tecnica "a scala di corda".

- da parte del paziente: importantissimo lavare le mani e il braccio dove è posizionata la FAVp;

- da parte dell'operatore: lavare le mani prima di toccare il braccio della FAVp del paziente e prima di iniziare la procedura di incannulazione.

La puntura della FAVp deve essere effettuata sempre con tecnica "a scala di corda" (Fig. 1). Tale tecnica si basa sulla variazione del sito di puntura lungo tutta la lunghezza della protesi. L'accortezza è quella di effettuare le punture rispettando una distanza di 3 centimetri dalle estremità della protesi, sia essa retta o a loop, per evitare eventuali danni alle anastomosi; altresì è necessario astenersi dal pungere la sezione preformata della protesi a loop (curvatura della protesi), per evitare 


\section{PUNTURA PROTESI}

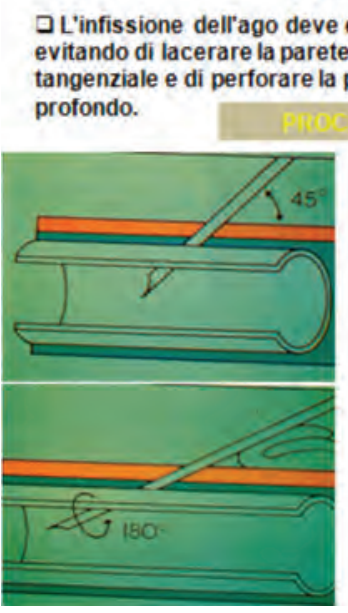

Inserire rago con la parte affilata posta anteriormente e con un angolo di $45^{\circ}$

$\square$ Una volta penetrati nel lume avanzare lentamente abbassando la coda dell'ago a $150^{\circ}$

Ruotare successivamente di $180^{\circ}$ per evitare la perforazione della parete protesica sottostante.
Fig. 2 - Angolazione dell'ago nella puntura della protesi (pubblicata con permesso da: Gessaroli M. Accessi vascolari per emodialisi (II ed.). Torino: Edizioni Minerva Medica; 2001, pag. 124) (3).

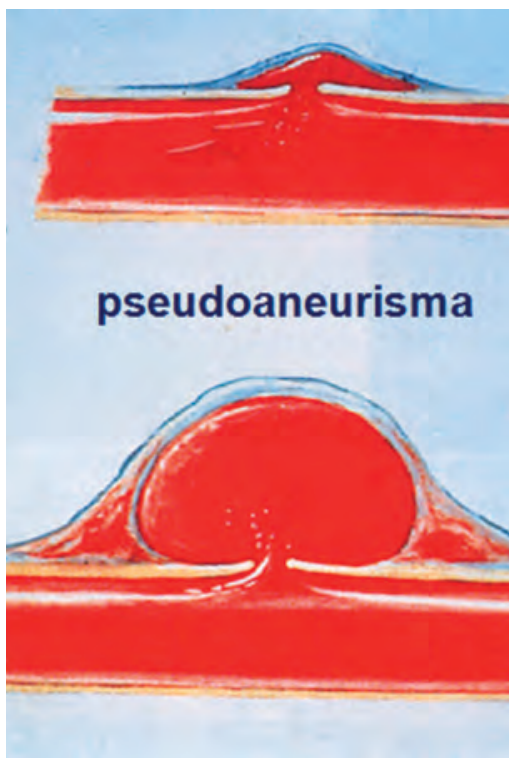

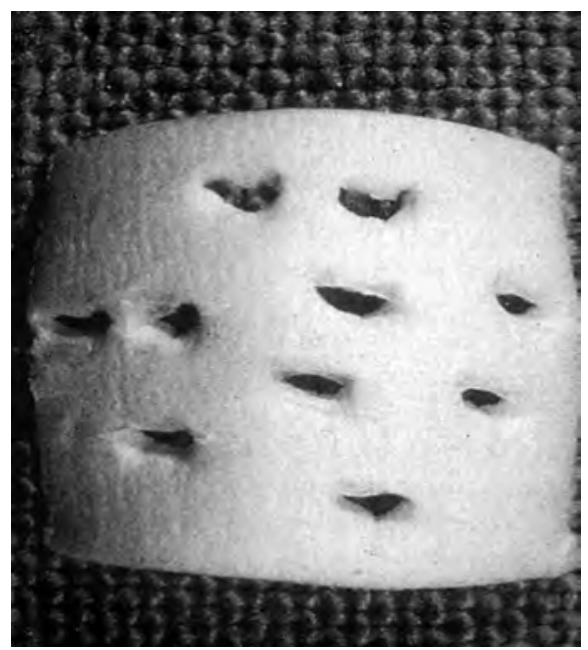

Fig. 3 - Perdita di tessuto protesico in sede di puntura. (pubblicata con permesso da: Gessaroli M. Accessi vascolari per emodialisi (II ed.). Torino: Edizioni Minerva Medica; 2001, pag. 125) (3).

di perforare la parete posteriore della protesi.

L'infissione dell'ago deve essere effettuata con un'adeguata angolatura, evitando di lacerare la parete anteriore in caso di inserimento troppo tangenziale, e di perforare la parete posteriore in caso di inserimento troppo profondo (Fig. 2), quindi progressione lenta dell'ago.

\section{Possibili complicanze}

Le comuni complicanze della FAVp sono riassunte nella Tabella III, e sono rappresentate dalle infezioni e dagli eventi trombotici dell'accesso vascolare. A differenza della FAVn, la formazione di pseuodaneurismi rappresenta un evento potenzialmente più frequente nella FAVp. A seguito di ripetute manovre di incannulazione della protesi, si formano nella sede di infissione degli aghi dei veri e propri fori nella parete (Fig. 3) che vengono tamponati da tessuto cicatriziale.

La ripetuta puntura della protesi nella stessa area genera un'ampia perdita di tessuto sintetico protesico che, se di dimensioni eccessive e/o non adeguatamente tamponato da tessuto cicatriziale, diventa sede di flusso sanguigno tra gli

TABELLA III - Comuni complicanze della FAV protesica

\begin{tabular}{lll}
\hline Tipo & Caratteristica & Provvedimento \\
\hline TROMBOSI & Assenza di fremito & Chiamare il medico per trombectomia \\
LESIONI CUTANEE & $\begin{array}{l}\text { Necrosi della cute sovrastante il sito di puntura per } \\
\text { punture zonali ripetute }\end{array}$ & Controllare che la protesi non sia esposta \\
& & Disinfezione locale \\
& Cute calda e arrossata, eventuale presenza di secrezioni & Evitare puntura \\
INFEZIONI & & Esame colturale \\
& & Disinfezione sistemica \\
& & Antibioticoterapia \\
PSEUDOANEURISMI & In sede della anastomosi arteriosa o più frequentemente & Evitare puntura \\
& lungo il decorso della protesi, nelle sedi di maggiore & Segnalare per revisione chirurgica Evitare \\
& utilizzo (punture troppo ravvicinate) & puntura \\
\hline
\end{tabular}


strati della protesi e può dare origine alla formazione di uno pseudoaneurisma (Fig. 4) che può essere scambiato per un ematoma, con la differenza che è rifornito continuamente dal sangue che passa nella protesi (3).

\section{Discussione}

La FAVp è un accesso vascolare che da un punto di vista chirurgico è di facile confezionamento, e da un punto di vista tecnico si può usare in tempi più brevi. Da un punto di vista infermieristico necessita di un'attenta sorveglianza rispetto alle complicanze trombotiche e infettive e di una definita manualità nell'inserimento degli aghi, da pungere mandatoriamente con tecnica a "scala di corda"; la puntura ad area è da evitare assolutamente. Per questi motivi espone potenzialmente il paziente a un maggiore dolore in fase di puntura. La valutazione della sofferenza di pazienti già compromessi fisicamente, fragili psicologicamente, con ridotte aspettative di vita e sottoposti a ripetute manovre invasive e dolorose viene tenuta in scarsa considerazione.

Avendo l'infermieristica come obiettivo la persona nel suo percorso di malattia/salute, questa "mancanza" di considerazione per gli elementi psico-fisici dinamici induce elementi di riflessione e sostiene la necessità di un'accurata valutazione dei pazienti idonei

\section{Conclusioni}

L'età anagrafica del paziente in emodialisi è in costante aumento e la scelta di quale accesso vascolare permanente realizzare rappresenta una sempre più frequente e importante problematica. Lo sviluppo nell'ultimo periodo di materiali biocompatibili resistenti a barotraumi e ripetute manovre di incannulazione ripropone il quesito sulla opportunità di utilizzare, invece di FAVn o CVC, accessi vascolari protesici. Le competenze infermieristiche devono comprendere abilità e conoscenze sulle tecniche di puntura e gestione specifiche delle fistole protesiche. Le FAVp possono essere una valida alternativa di efficienza clinica e migliore qualità di vita in pazienti selezionati, se allestite correttamente, gestite con adeguati accorgimenti tecnici e competenze assistenziali, in contesti in cui vi sia la disponibilità per interventi immediati in caso di eventi avversi.

"... bisogna avere la forza di non trattare i pazienti come casi e di non rendere la cura della malattia più PESANTE della malattia stessa..." (Sir Robert Hutchinson) (4).

\section{Disclosures}

Financial support: No financial support was received for this submission. Conflict of interest: The author has no conflict of interest.

\section{Bibliografia}

1. Tazza L, Galli F, Mandolfo S, et al; PP1SIN Study Investigators. [Indications for vascular grafts as dialysis access; consensus from the Italian experience]. G Ital Nefrol. 2010;27(5):512-21.

2. National Kidney Foundation. 2006 Updates Clinical Practice Guidelines and Recommendations. Hemodialysis Adequacy, Peritoneal Dialysis Adequac. Vascular Access. 2006. Access, Guide Line 3, Cannulation of Fistula and Graft, 2006, pag 261-270 .

3. Gessaroli M. Accessi vascolari per emodialisi (II ed.). Torino: Edizioni Minerva Medica; 2001.

4. Beltrutti D, Brena SF, Tiengo M. Dolore ed energia. Fidenza: Mattioli 1885, 2004:1-222. 